Caries Res. 1988;22:64

\title{
Acknowledgements to Referees
}

To the same extent as authors submitting good manuscripts, the scientific standing of Caries Research is kept high by the untiring

anonymous services of numerous referees whose expert advice is highly appreciated. K.G.

König and A.J.M. Plasschaert

J. Afseth

J.K. Ainamo

RJ. Anderson

B. Angmar-Månsson

J. Arends

R.E. Baier

B. Bibby

R.J. Billings

J. Birkeland

D. Birkhed

J. de Boever

J.J. ten Bosch

A. Boyde

G.H.W. Bowden

W. Bowen

P. Brandtzaeg D. Bratthall A.L.J.J. Bronckers

C. Bruun

J.J. Burckhardt J. Carlos J. Carlsson V. Caslavska N.W. Chilton M.E.J. Curzon C. Dawes D.P. DePaola G.H. Dibdin W. Distler M.C. Downer F.C.M. Driessens J.W.E. van Dijk A.G. Dijkman J.E. Eastoe W.M.Edgar S. Edwardsson A.D. Eisenberg J. Ekstrand

J.C. Elliott Th. Ericson J.D.B. Featherstone F.R. von der Fehr O. Fejerskov A.R. Firestone R.

Frank R.J. Gibbons P.E. Gjermo P.O. Glantz R.L. Glass H. Graf

EJ.’s-Gravenmade R.M. Green T.H. Grenby A. Groeneveld P. Gron A.I. Hamilton J.L.

Hardwick D.I. Hay

A. Hefti

M.J. Hicks

J.S. van der Hoeven

P.J. Holloway

P. Hoppenbrouwers

P. Hotz

J. van Houte

B. Houwink

T. Imfeld

G.S. Ingram

G.N. Jekins 
M.Jensen

N.W.Johnson

M.H. deJong

S. Joyston-Bechal H. Kalsbeek S. Kashket E.A.M. Kidd

M. Kilian

E. Kirkegaard

I. Kleinberg

T. Koulourides W. Künzel

F. Lagerlöf

K.P. Lang

S.A. Leach

T. Lehner

A. Linde

H. Löe

W.J. Loesche H. Luoma K.K. Mäkinen H. Margolis P.D. Marsh T.M. Marthaler B.C. McBride J.R. Mellberg L. Mikkelsen F.H.M. Mikx E.C. Moreno S. Mundorff J.J. Murray J.M. Navia M.N. Naylor E. Newbrun H. Newesely H.N. Newman R. Nivard G.S. Nixon J. Norén

B. Nyvad

D.M.O’Mullane

E.I.F. Pearce

S. Poulsen

DJ. Purdell-Lewis

H.R. Rawls

D.H. Retief C. Robinson A.H. Rogers G. Rølla A.J. Rugg-Gunn M.J.M. Schaeken A.A. Scheie R. Schmid H.E.Schroeder L. Seppå I.L. Shannon L.M. Silverstone P.F. van der Stelt K.W.

Stephen G.K. Stookey J.D. de Stoppelaar M.Svanberg J.M. Tanzer A. Tatevossian J.O. Tenovuo E. Theilade

A. Thylstrup

M. Triller

G.J. Truin

P.A. Vingerling

W. Visser

F. Vogels

B.J. Wagg

G.J. Walker

J.A. Weatherell

A.H. Weerkamp

J.S. Wefel

G.M. Whitford

J.M.Wilton

G.B. Winter 